\title{
Gamma rays and neutrinos from dark matter annihilation in galaxy clusters
}

\author{
Qiang Yuan ${ }^{1}$, Peng-Fei Yin ${ }^{2}$, Xiao-Jun Bi ${ }^{1,3}$, Xin-Min Zhang ${ }^{4}$, and Shou-Hua Zhu ${ }^{2,3}$ \\ ${ }^{1}$ Key Laboratory of Particle Astrophysics, Institute of High Energy Physics, \\ Chinese Academy of Sciences, Beijing 100049, P. R. China \\ 2 Institute of Theoretical Physics 85 State Key Laboratory of Nuclear Physics and Technology, \\ Peking University, Beijing 100871, P.R. China \\ 3 Center for High Energy Physics, Peking University, Beijing 100871, P.R. China \\ 4 Theoretical Division, Institute of High Energy Physics, \\ Chinese Academy of Sciences, Beijing 100049, P. R. China
}

(Dated: November 5, 2018)

\begin{abstract}
The $\gamma$-ray and neutrino emissions from dark matter (DM) annihilation in galaxy clusters are studied. After about one year operation of Fermi-LAT, several nearby clusters are reported with stringent upper limits of $\mathrm{GeV} \gamma$-ray emission. We use the Fermi-LAT upper limits of these clusters to constrain the DM model parameters. We find that the DM model distributed with substructures predicted in cold DM $(\mathrm{CDM})$ scenario is strongly constrained by Fermi-LAT $\gamma$-ray data. Especially for the leptonic annihilation scenario which may account for the $e^{ \pm}$excesses discovered by PAMELA/Fermi-LAT/HESS, the constraint on the minimum mass of substructures is of the level $10^{2}-10^{3} \mathrm{M}_{\odot}$, which is much larger than that expected in CDM picture, but is consistent with a warm DM scenario. We further investigate the sensitivity of neutrino detections of the clusters by IceCube. It is found that neutrino detection is much more difficult than $\gamma$-rays. Only for very heavy DM $(\sim 10 \mathrm{TeV})$ together with a considerable branching ratio to line neutrinos the neutrino sensitivity is comparable with that of $\gamma$-rays.
\end{abstract}

PACS numbers: 95.35.+d,95.85.Pw,95.85.Ry,98.65.Cw 


\section{INTRODUCTION}

The existence of dark matter (DM) has been established by many astrophysical observations, but the nature of DM paticle is still unclear. Among the large amount of candidates proposed in many theories of new physics, the weakly interacting massive particle (WIMP) is the most popular and attractive one [1, 2]. The mass of WIMP is generally from a few $\mathrm{GeV}$ to $\mathrm{TeV}$, and the interaction strength is of the weak scale, which can give the right relic density of DM. In this scenario, the weak interaction of DM particles would produce observable standard model particles, such as charged anti-matter particles, photons and neutrinos. Investigating such particles from the cosmic rays (CRs) is the task of DM indirect detection.

The recently reported new signatures of CR positrons, antiprotons and electrons by PAMELA [3, 4], ATIC [5], HESS [6, 7] and Fermi-LAT [8] have stimulated great interests and extensive studies of the DM indirect searches. The DM scenario with mass $O(\mathrm{TeV})$, leptonic annihilation/decay final states and a high annihilation/decay rate can well explain the observational data (e.g., 9 , 10]). Furthermore, more quantitative constraints on the DM model parameters can be derived through a global fitting method [11, 12].

Regardless of detailed models of DM to explain the data, it is essential to find observable signals to test the models. Since the charged particles will gyrate in the magnetic field and lose most of the source information, it is difficult to test the DM models using only the data of charged CRs. Gamma-rays and neutrinos seem to be very good probes. There are several advantages of using $\gamma$-ray photons and neutrinos to investigate the DM models. Firstly, photons and neutrinos propagate along straight line and can trace back to the source sites where the DM annihilation/decay takes place. Secondly there is little interaction during the propagation and most of the primary source information hold. Thirdly the effective volume of which photons and neutrinos can reach is much larger than that of charged particles, e.g., from the Milky Way to extragalactic space, and even the early Universe. It has been shown in some works that $\gamma$-rays and neutrinos can be powerful tools to test the DM scenarios explaining the CR lepton data (e.g., [13 20], [21 27]).

There are many sites proposed to be good candidates for the search of $\gamma$-rays and neutrinos from DM, such as the Galactic center [13 15], Galactic halo [16, 20, 28], satellite galaxies

or substructures [29 32], the extragalactic space [33 35] and the emissions at the early Universe [36 38]. As the largest gravitational bounding system in the Universe, galaxy 
clusters may also be useful for DM indirect searches [39]. Pinzke et al. investigated the $\gamma$-ray emission from nearby clusters and used EGRET upper limits to set constraints on the DM model parameters [40]. They found that if the DM annihilation was responsible for the electron/positron excesses and the luminosity-mass distribution of DM substructures in clusters followed the extrapolation of numerical simulation results, the minimum mass of DM subhalos should be larger than $10^{-2} \mathrm{M}_{\odot}$ in order not to exceed the EGRET limits. This is a useful way to study the particle nature of DM through structures.

After more than one year's operation, Fermi-LAT reported some results about the $\gamma$-ray emission from galaxy clusters [41]. Non detection of significant $\gamma$-ray emission from galaxy clusters was reported except for Perseus cluster, in which the emission from the central galaxy NGC 1257 was discovered [42]. The upper limits given by Fermi-LAT are lower by more than one order of magnitude than that given by EGRET. It can be expected that the new results from Fermi-LAT will set much stronger constraints on the DM models. In Ref. [41] the constraints on DM mass and annihilation cross section were presented assuming $\mu^{+} \mu^{-}$and $b \bar{b}$ channels. In this work we will also use the Fermi-LAT upper limits to constrain DM model parameters. Different from Ref. [41], we will pay more attention on the implication of DM structure properties such as the minimal mass of subhalo $M_{\min }$, which would be important for understanding the nature of DM particle. This is one of the motivations of this study.

Another motivation of this work is the neutrino emission. Neutrinos can be served as an independent diagnostic of DM indirect searches besides photons. It has been shown that the measured atmospheric neutrino background can set effective constraints on the DM annihilation cross section [43, 44]. There are no high energy astrophysical neutrinos being detected currently, so it is valuable to explore the sensitivity of the forthcoming neutrino detectors to the neutrino signals from the DM annihilation. Due to the very weak interaction cross section between neutrinos and matter, we generally need large detector volume. The ongoing experiment IceCube has an effective volume $\sim \mathrm{km}^{3}$, which would give unprecedented sensitivity for the neutrino detection up to very high energies. The detectability of neutrino signals from DM annihilation in galaxy clusters by e.g. IceCube, will be discussed in this work.

This paper is organized as follows. In Sec. II, we discuss the $\gamma$-ray emission from several galaxy clusters, and employ the recent Fermi-LAT limits of these clusers to constrain the DM 
model parameters. In Sec. III, we discuss the detectability of neutrino emission from the galaxy clusters by the neutrino detectors. The last section is our conclusions and discussions.

\section{GAMMA RAYS FROM GALAXY CLUSTERS}

\section{A. Cluster sample}

It is known that the objects with high masses and small distances will be very efficient for the DM searches. Therefore nearby massive clusters are the first choice of study. Here we adopt a sample of 6 clusters with redshift from 0.0031 to 0.0231 (corresponding to distance from 13 to $100 \mathrm{Mpc}$ for a standard $\Lambda \mathrm{CDM}$ cosmology), which are reported with flux upper limits by Fermi-LAT. The basic parameters of these clusters are compiled in Table प

The flux of DM annihilation from cluster is generally scaled with $M_{200}^{\alpha} / d^{2}$, where $\alpha$ depends on the concentration-mass relation and DM profile of the halo. In this work we will assume NFW profile for the halo of cluster. The final results are expected not sensitively dependent on the profile since most of the cluster lies in the angular window of Fermi-LAT (3.5 for $100 \mathrm{MeV},[45])$. As a benchmark configuration, we adopt the concentration-mass relation fitted from X-ray observations [46]

$$
c_{\mathrm{vir}}=\frac{9.0}{1+z} \times\left(\frac{M_{\mathrm{vir}}}{10^{14} h^{-1} \mathrm{M}_{\odot}}\right)^{-0.172} .
$$

After correcting the definition of virial overdensity in Ref. [46] $(\Delta \approx 100)$ to $\Delta=200$ we have [47]

$$
c_{200}=\frac{6.9}{1+z} \times\left(\frac{M_{200}}{10^{14} \mathrm{M}_{\odot}}\right)^{-0.178} .
$$

For this concentration-mass relation we find $\alpha \approx 0.65$. Comparing the quantity $M_{200}^{\alpha} / d^{2}$ among these clusters, we find that DM signals from clusters NGC 4636, M49 and Fornax are of the same level, and are several times larger than the rest three clusters. In the following we will see that these three clusters will indeed give stronger constraints on the DM models.

\section{B. Gamma-ray emission from DM distribution in clusters}

There are generally two kinds of $\gamma$-ray emission from DM annihilation: one is produced directly from the annihilation final state particles which is called primary emission (such as 
TABLE I: Cluster sample

\begin{tabular}{cccccc}
\hline \hline Name & $z^{a}$ & R.A. $^{a}$ & Dec. $^{a}$ & $M_{200}\left(10^{14} \mathrm{M}_{\odot}\right)^{b}$ & $r_{200}(\mathrm{Mpc})^{b}$ \\
\hline NGC 4636 & 0.0031 & $12^{h} 43^{m}$ & $2^{\circ} 41^{\prime}$ & 0.25 & 0.60 \\
M49 & 0.0033 & $12^{h} 30^{m}$ & $8^{\circ} 00^{\prime}$ & 0.46 & 0.73 \\
Fornax & 0.0046 & $03^{h} 39^{m}$ & $-35^{\circ} 27^{\prime}$ & 1.00 & 0.95 \\
Centaurus & 0.0114 & $12^{h} 49^{m}$ & $-41^{\circ} 18^{\prime}$ & 2.66 & 1.32 \\
AWM 7 & 0.0172 & $02^{h} 55^{m}$ & $41^{\circ} 35^{\prime}$ & 4.28 & 1.54 \\
Coma & 0.0231 & $13^{h} 00^{m}$ & $27^{\circ} 59^{\prime}$ & 13.65 & 2.27 \\
\hline \hline
\end{tabular}

${ }^{a}$ Redshift and coordinates are adopted from NASA/IPAC Extragalactic Database, http://nedwww.ipac.caltech.edu/

${ }^{b}$ Virial mass and radius parameters are taken from Ref. [48].

the $\gamma$ rays by $\pi^{0}$ decay after hadronization or emission directly from final charged leptons), and the other is produced through interactions of final state particles with external medium or radiation field such as the inverse Compton (IC) radiation which is called secondary emission hereafter. The primary $\gamma$-ray flux observed on the Earth from DM annihilation in a galaxy cluster can be expressed as

$$
\phi^{p r i}=\frac{\langle\sigma v\rangle}{2 m_{\chi}^{2}} \frac{\mathrm{d} N}{\mathrm{~d} E} \times \frac{\int \rho^{2}(r) \mathrm{d} V}{4 \pi d_{L}^{2}},
$$

where $m_{\chi}$ is the mass of DM particle, $\langle\sigma v\rangle$ is the annihilation cross section of DM, $\frac{\mathrm{d} N}{\mathrm{~d} E}$ is the yield spectrum of $\gamma$-rays per annihilation which is simulated using PYTHIA [49], $d_{L}$ is the luminosity distance of the cluster, $\rho(r)$ is the density distribution of DM inside the cluster with $r$ the distance from the cluster center. All of the cluster is taken into account in the integral since the analysis of Fermi-LAT was done in a 10 degree radius of each cluster [41], which is large enough to contain the whole cluster halo. For the smooth halo we assume the density distribution to be NFW profile [50]

$$
\rho_{\mathrm{sm}}(r)=\frac{\rho_{s}}{\left(r / r_{s}\right)\left(1+r / r_{s}\right)^{2}}
$$

where parameters $r_{s}$ and $\rho_{s}$ can be determined by the concentration-mass relation and normalization of total mass.

Since there are substructures in the clusters, such as galaxy groups and galaxies, we have to take these into account. We will see later that the existence of substructures enhances 
the annihilation luminosity of DM and is the main reason that affects the $\gamma$ flux. To take into account the effect of substructures, we replace $\rho^{2}$ in Eq. (3) with $\rho_{\text {tot }}^{2} \equiv \rho_{\mathrm{sm}}^{2}+\left\langle\rho_{\mathrm{sub}}^{2}\right\rangle$, where the average density square of substructures reads

$$
\left\langle\rho_{\mathrm{sub}}^{2}(r)\right\rangle=\int \mathrm{d} M \frac{\mathrm{d} N}{\mathrm{~d} V \mathrm{~d} M} \times L(M)
$$

in which $\frac{\mathrm{d} N}{\mathrm{~d} V \mathrm{~d} M}$ is the number density of subhalos in mass bin $\mathrm{d} M, L(M)=\int_{V_{\text {sub }}} \rho_{\text {sub }}^{2} \mathrm{~d} V^{\prime}$ is the intrinsic annihilation luminosity of a subhalo with mass $M$. In this work we will employ the results from recent high resolution simulation, Aquarius [51, 52] to treat the subhalos. Because the concentration and density profile of subhalos are very complicated inside the host halo, the detailed computation using Eq. (5) is difficult. Thus we directly adopt the counted results of luminosity distribution from the simulation ${ }^{1}$ [53]

$$
\frac{\mathrm{d} \mathcal{L}}{\mathrm{d} M}(r, M) \equiv \frac{\mathrm{d} N}{\mathrm{~d} V \mathrm{~d} M} \times L(M) \propto\left(\frac{r}{0.2 r_{200}}\right)^{-0.1}\left(1+\frac{r}{0.2 r_{200}}\right)^{-2.9} \times M^{-1.16}
$$

Similar with Ref. [40] we adopt a scale between the Milky Way like halo given in Aquarius simulation and the case of clusters, i.e., the ratio of $\mathcal{L}_{\text {sub }} / \mathcal{L}_{\text {sm }}$ keeps unchanged whatever the mass is. The maximum mass of subhalos found in simulation is about $0.01 M_{\text {host }}$. But the minimum mass is not well known due to the limit of resolution of the numerical simulation. From the observational point of view, we have observed DM halos with mass $\sim 10^{7} \mathrm{M}_{\odot}$, e.g. dwarf galaxies. While the study of free streaming of cold DM (CDM) particles indicates a minimum halo mass down to $\sim 10^{-7} \mathrm{M}_{\odot}\left[54\right.$ ]. In this work we leave $M_{\min }$ to be a free parameter and investigate the effects of $M_{\min }$ on the DM signals.

Besides the primary $\gamma$-ray emission, there is also secondary production of $\gamma$-ray photons through the IC scatterings between the DM induced electrons/positrons and the cosmic microwave background (CMB) field. For the calculation of the fluxes of secondary IC emission please see the Ref. [16]. Note that when calculating the energy loss rate of electrons/positrons, both the IC loss induced by scattering with CMB photons and the synchrotron loss in the magnetic field in clusters are considered. The average value of magnetic field strength is assumed to be $\sim 1 \mu \mathrm{G}[55]$.

\footnotetext{
${ }^{1}$ This relation is different from that given in Ref. [51], where $L(>M) \approx M^{-0.226}$ was found. According to this fit we have $L(>M) \approx\left(M^{-0.16}-M_{\max }^{-0.16}\right)$, which gives similar behavior as that in Ref. [51] in the large (resolved) mass range of the simulation, but is different when extrapolating to low (unresolved) mass range.
} 

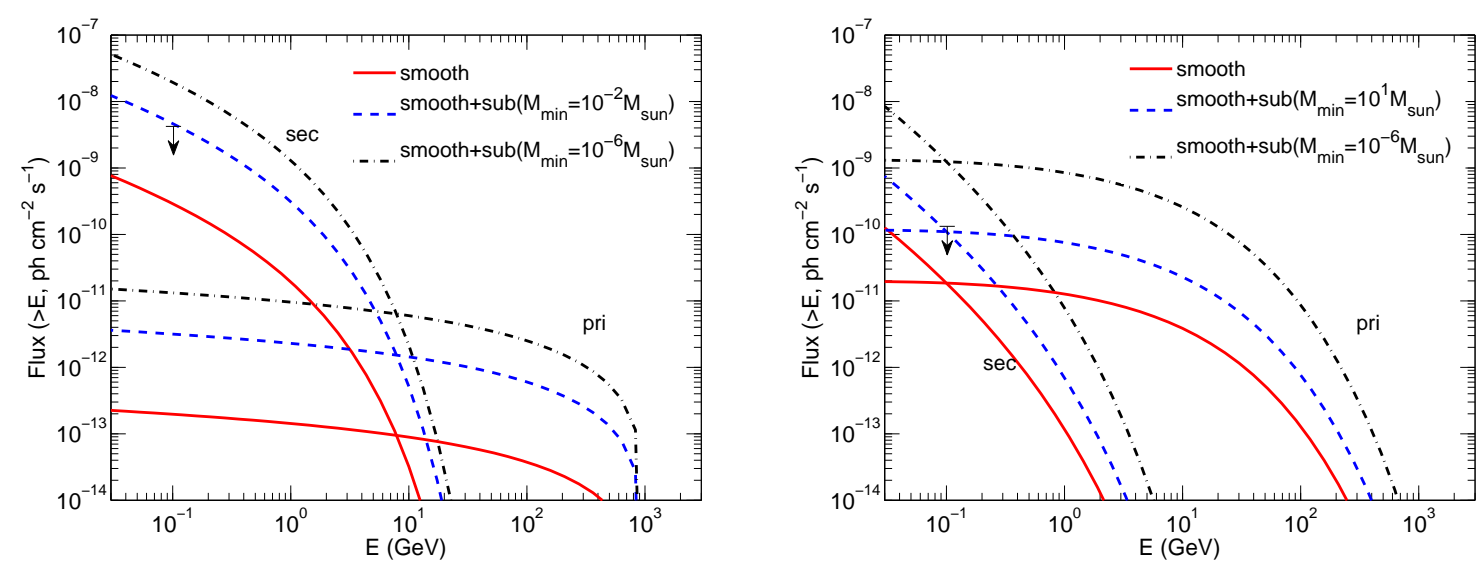

FIG. 1: Integral spectra of the IC and FSR components from DM annihilation in Fornax cluster. The left panel is for $\mu^{+} \mu^{-}$channel, and the right panel is for $b \bar{b}$ channel respectively. Also shown are the $95 \%$ upper limits (arrows) of Fermi-LAT 1 yr observations. See the text for details.

For illustration we show the integral spectra of $\gamma$-rays from DM annihilation in Fornax cluster in Fig. 1. Here we adopt a sample DM model with $m_{\chi}=1 \mathrm{TeV},\langle\sigma v\rangle=10^{-23}$ $\mathrm{cm}^{3} \mathrm{~s}^{-1}$, and the annihilation channels with $\mu^{+} \mu^{-}$(left) and $b \bar{b}$ (right) respectively. The primary and secondary components are shown separately. In each group we show three curves which represent the smooth halo contribution, the total emission with subhalos down to two different $M_{\min }$. The $95 \%$ confidence level upper limits from Fermi-LAT are shown by arrows. It is shown that in the energy range interested here, i.e. $0.1-10 \mathrm{GeV}$, the secondary radiation from IC is dominant for $\mu^{+} \mu^{-}$channel, while for $b \bar{b}$ channel the primary contribution is dominant. This is because for $\mu^{+} \mu^{-}$final state the spectra of photons and electrons are relatively hard, and the secondary produced photons through IC can just lie in the interested energy range. For $b \bar{b}$ final state the energies of photons and electrons from the hadronic cascade are generally much lower than the mass of DM, so the IC component dominates at even lower energies $(<0.1 \mathrm{GeV})$. This conclusion will always hold for $m_{\chi}$ ranging from $100 \mathrm{GeV}$ to $10 \mathrm{TeV}$ for $\mu^{+} \mu^{-}$channel $(10 \mathrm{GeV}$ to $1 \mathrm{TeV}$ for $b \bar{b})$, which is the sensitive region explored by Fermi-LAT.

In [41] the Fermi-LAT has derived preliminary constraints on the $\gamma$-ray fluxes from DM annihilation from these clusters by assuming the $\mu^{+} \mu^{-}$and $b \bar{b}$ final states. According to these results, we derive the constraints on the $m_{\chi}-\langle\sigma v\rangle$ plane. In Figs. 2 we show the constraints for the $\mu^{+} \mu^{-}$channel. It is shown that the constrains of the smooth component 

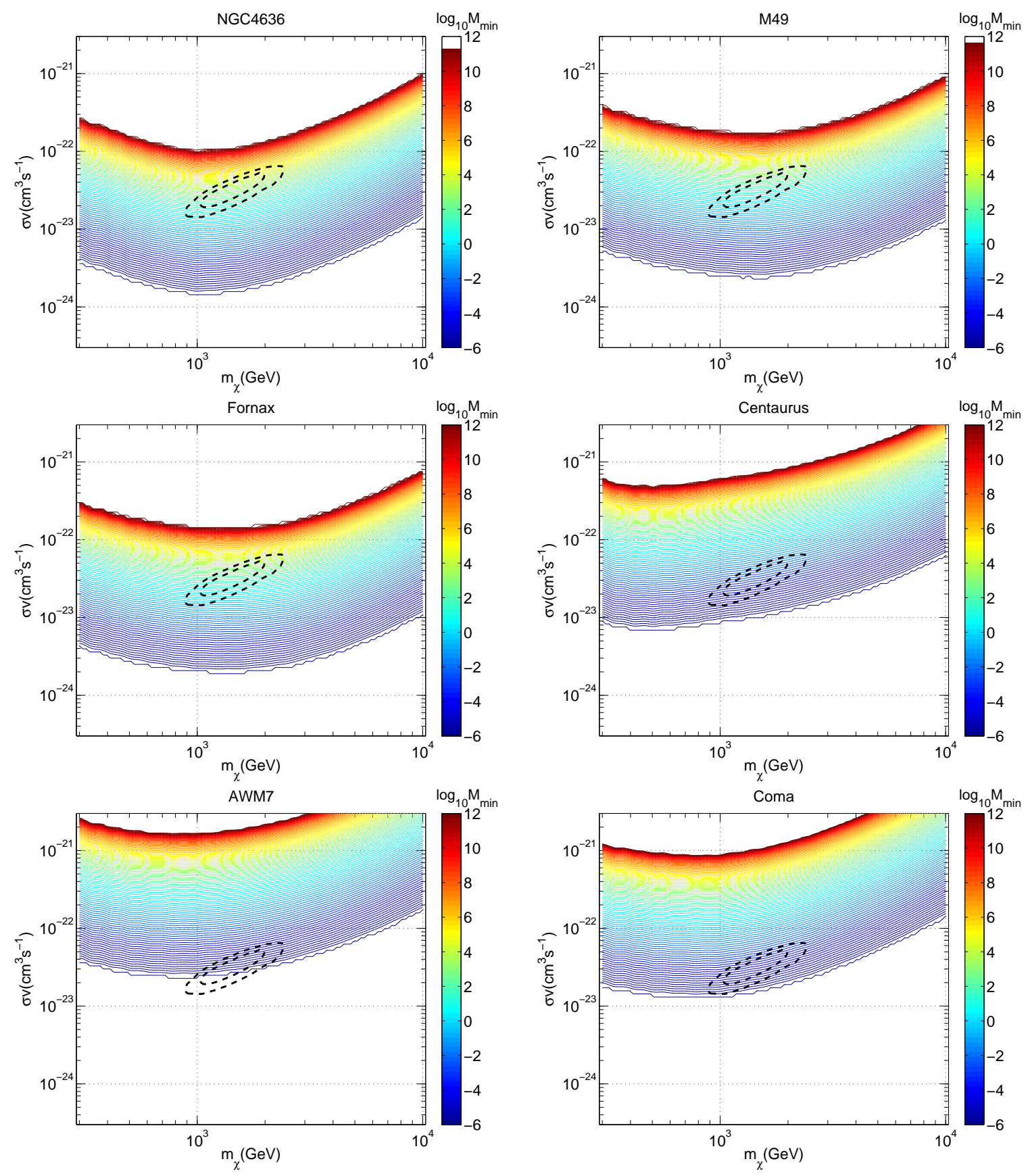

FIG. 2: Fermi-LAT constraints on the DM model parameters $m_{\chi}$ and $\langle\sigma v\rangle$ for different minimum halo mass $M_{\min }$. The DM annihilation channel is $\mu^{+} \mu^{-}$. Dashed circles are the $3 \sigma$ and $5 \sigma$ parameters regions which can fit the PAMELA/Fermi-LAT/HESS data of the CR positrons/electrons [56].

(that $M_{\min }=10^{12} M_{\odot}$ ) is still very weak. If the substructures are taken into account the constraints can be stronger by more than one order of magnitude, depending on the free 

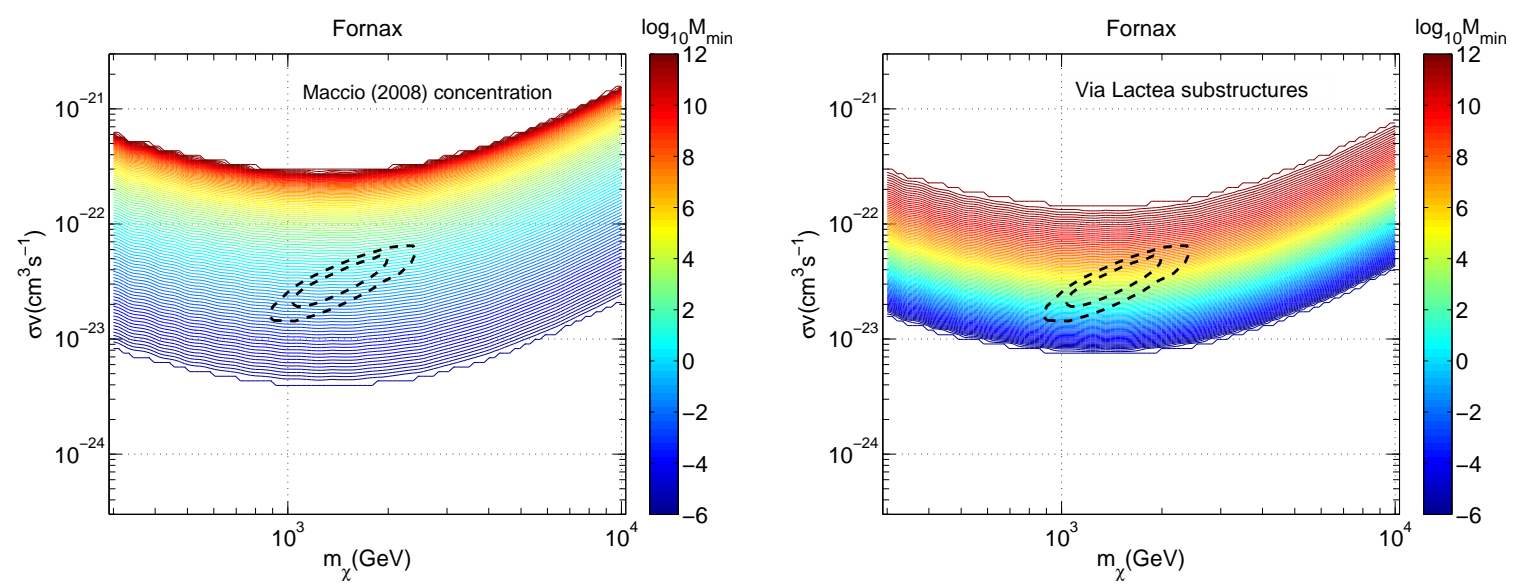

FIG. 3: Comparison of the constraints for Maccio et al. (2008) concentration model (left, [57]) and Via Lactea substructure model (right, [58]).

streaming mass of DM subhalo. If the minimum mass of DM subhalos can be as low as $10^{-7}-10^{-6} \mathrm{M}_{\odot}$ as predicted in the supersymmetric DM scenario, the constraint on the cross section of TeV DM can reach $\sim 10^{-24} \mathrm{~cm}^{3} \mathrm{~s}^{-1}$. This constraint is actually powerful enough to explore the DM models which are proposed to explain the CR lepton excesses. In Fig. 2 we plot the favored parameter regions of the DM model to explain the PAMELA/FermiLAT/HESS data of the CR positrons/electrons with $\mu^{+} \mu^{-}$final state [56]. We can see that for almost all of the 6 clusters the PAMELA/Fermi-LAT/HESS favored parameter regions can be excluded if $M_{\text {min }}$ is down to $\sim 10^{-6} \mathrm{M}_{\odot}$. If $\mathrm{DM}$ annihilation is responsible for the observational positron/electron excesses, it may indicate that the cutoff of $M_{\min }$ should be much larger [40]. For clusters Fornax and NGC 4636, the constraint of $M_{\min }$ is about $10^{2}-10^{3} \mathrm{M}_{\odot}$ for the best fit mass and cross section ${ }^{2}$. This constraint is much stronger than that derived using EGRET upper limit about Virgo cluster [40].

This cutoff of the mass of substructures may have important implication of the particle nature of DM. It gives an estimate of the free streaming scale of the matter power spectrum as $k<750 \mathrm{Mpc}^{-1}$, which is not very far from the lower limits given by Lyman- $\alpha$ power spectrum measurements [61, 62]. Compared with the canonical value expected in CDM

\footnotetext{
${ }^{2}$ Note that here we assume a constant boost factor of the DM models responsible to the electron/positron excesses. However, as proposed in the literature the large boost factor might be due to velocity-dependent annihilation cross section such as the Sommerfeld effect [59, 60]. In that case the boost factor might be different in clusters from in the Galaxy. We do not discuss this effect in detail in this study.
} 

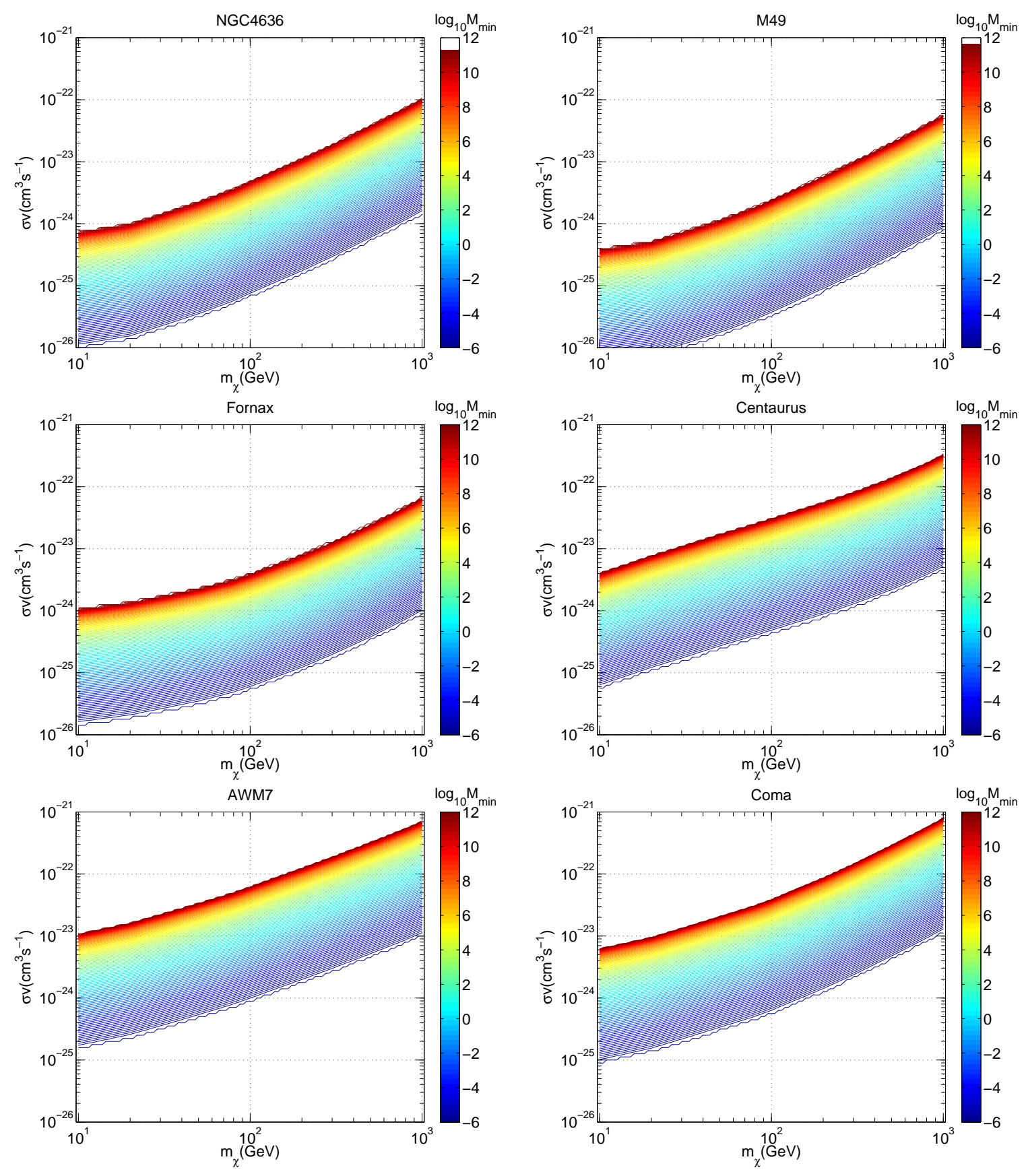

FIG. 4: The same as Fig. 2 but for $b \bar{b}$ final state of DM annihilation.

picture, it favors a warm massive DM scenario which may be produced non-thermally in the early Universe [63]. Such nonthermally produced DM particles have large initial velocity and large free streaming. Thus the matter power spectrum is suppressed at small scales and leads to less low mass subhalos [64, 65].

As a check of the model uncertainties of the halo structure configuration, we compare 
the results using Maccio (2008) concentration-mass relation given in Ref. [57]

$$
c_{200}=\frac{3.56}{1+z} \times\left(\frac{M_{200}}{10^{15} \mathrm{M}_{\odot}}\right)^{-0.098} .
$$

For substructures we also use the results from another high-resolution simulation Via Lactea. According to Fig. 3 of Ref. [58], the substructure enhancement is simply extracted to be $B=10 \times\left(M_{\min }^{-0.048}-M_{\max }^{-0.048}\right)$ with $M_{\max }=0.01 M_{\text {host }}$. Note that there is a host halo mass dependence of $B$ as given in Ref. [58]. However, since the results with $M_{\text {host }}$ larger than the galaxy scale halo were not calibrated in the simulation, we adopt $B\left(M_{\text {host }}\right)$ at $M_{\text {host }}=10^{12}$ $\mathrm{M}_{\odot}$ and apply it to the cluster scale halos. This treatment is also consistent with the above assumption that we adopt the scaling relation of Eq. (6) to be the same for Milky Way halo and cluster halos. The extrapolation to the cluster masses following the $M_{\text {host }}$ dependence of $B$ in Ref. [58] would lead to a two times larger boost factor. The constraints for $\mu^{+} \mu^{-}$channel from Fornax cluster for these two models are shown in Fig. 3. It is shown that for Maccio (2008) concentration the constraint is about 2 times weaker than the benchmark model. And for Via Lactea substructures the total substructure enhancement is some weaker than that of Aquarius simulation. However, in both of these cases we see that the PAMELA/Fermi-LAT/HESS favored parameter regions can be constrained, given $M_{\text {min }} \sim 10^{-6} \mathrm{M}_{\odot}$. For the best fit mass and cross section the constraint on $M_{\min }$ is about $10^{0}-10^{2} \mathrm{M}_{\odot}$.

The results for $b \bar{b}$ channel are shown in Fig. 4. We can see that for $m_{\chi} \approx 100 \mathrm{GeV}$ the most stringent constraint of $\langle\sigma v\rangle$ can reach the thermal scale of $3 \times 10^{-26} \mathrm{~cm}^{3} \mathrm{~s}^{-1}$ for $M_{\text {min }}=10^{-6} \mathrm{M}_{\odot}$. The future observation of Fermi-LAT can put a strong constraint on the DM model even for the thermal scenario. Note that for $b \bar{b}$ channel only the primary emission is assumed when deriving the flux limits. If the IC component is included the constraints will be stronger for relatively heavy DM mass $(\sim \mathrm{TeV})$. Finally we should point out that $b \bar{b}$ channel is typically not suitable to explain the lepton excesses observed by PAMELA/FermiLAT/HESS, due to both the constraint from PAMELA $\bar{p} / p$ data [4, 9] and the spectral shape required by lepton excesses [10]. Here we include the study of $b \bar{b}$ channel is just to show the power of Fermi-LAT to the supersymmetric-like DM particles. 


\section{NEUTRINOS}

In this section, we discuss the sensitivity of detecting neutrino signals from clusters. The cluster could be treated as high energy neutrino point source, and it is possible to be observed at the on-going large volume neutrino telescopes, such as IceCube. To suppress the large atmospheric muon background, the neutrino telescopes usually detect the upward muons induced by muon neutrinos through interacting with the matter surrounding the detectors. Therefore, the south pole based detector IceCube is more suitable to probe the neutrino sources in the northern hemisphere. For the sample of clusters in Table I, Fornax and Centaurus locating in the southern hemisphere are not good candidates of Icecube ${ }^{3}$. AWM 7 with declination of $41^{\circ} 35^{\prime}$ and Coma with declination of $27^{\circ} 59^{\prime}$ are suitable for IceCube, but such two clusters are more distant away from us than other clusters. Taking into account the location, mass and distance, we find M49 with declination of $8^{\circ} 00^{\prime}$ is better to be detected than others.

Similar to the primary $\gamma$-ray flux, the neutrino flux from DM annihilation in the cluster can also be calculated according to Eq. (3), by replacing $\left.\frac{\mathrm{d} N}{\mathrm{~d} E}\right|_{\gamma}$ with $\left.\frac{\mathrm{d} N}{\mathrm{~d} E}\right|_{\nu}$. In the following we will mainly discuss two DM annihilation channels, $\mu^{+} \mu^{-}$and $\mu^{+} \mu^{-}+\nu_{\mu} \bar{\nu}_{\mu}$. The $b \bar{b}$ channel as discussed in the previous section will also be mentioned. However, as we will see below, it gives negligible neutrino signals. We use the PYTHIA [49] to simulate the initial neutrino spectra from decay of annihilation final states. We further assume the neutrino flavor distribution is $1: 1: 1$ at the Earth due to vacuum oscillation during the propagation.

The through-going upward muon rate at the detector can be calculated as

$$
\frac{d N_{\mu}}{d E_{\mu}}=\int d \Omega \int_{E_{\mu}}^{m_{\chi}} d E_{\nu_{\mu}} \frac{d N_{\nu_{\mu}}}{d E_{\nu_{\mu}}}\left[\frac{d \sigma_{\mathrm{CC}}^{\nu p}\left(E_{\nu_{\mu}}, E_{\mu}^{0}\right)}{d E_{\mu}^{0}} n_{p}+(p \rightarrow n)\right] R\left(E_{\mu}\right)+(\nu \rightarrow \bar{\nu}),
$$

where $n_{p}\left(n_{n}\right)$ is the number density of protons (neutrons) in the matter around the detector, $R\left(E_{\mu}\right)$ named muon range is the distance that a muon can travel in matter before its energy drops below the threshold energy of detector $E_{\mathrm{th}}$, which is given by

$$
R\left(E_{\mu}\right)=\frac{1}{\rho \beta} \ln \frac{\alpha+\beta E_{\mu}}{\alpha+\beta E_{\mathrm{th}}},
$$

\footnotetext{
${ }^{3}$ The IceCube + DeepCore has the capability to search the downward neutrinos, but the angular resolution is fairly bad [66]. It is very difficult to distinguish the high energy neutrino sources from the high atmospheric neutrino background without powerful angular resolution.
} 
with $\alpha, \beta$ the parameters describing the energy loss of muons as $d E_{\mu} / d x=-\alpha-\beta E_{\mu}$.

The main background for high energy neutrino detection is the atmospheric neutrinos. The atmospheric neutrino flux decreases rapidly as energy increasing. We use a parametrization of atmospheric neutrino flux [67] which describes the results of Ref. [68] as

$$
\frac{\mathrm{d} N_{\nu}}{\mathrm{d} E_{\nu} \mathrm{d} \Omega}=N_{0} E_{\nu}^{-2.74}\left(\frac{0.018}{1+0.024 E_{\nu}|\cos \theta|}+\frac{0.0069}{1+0.00139 E_{\nu}|\cos \theta|}\right)
$$

where $N_{0}=1.95 \times 10^{17}\left(1.35 \times 10^{17}\right) \mathrm{GeV}^{-1} \mathrm{~km}^{-2} \mathrm{yr}^{-1} \mathrm{sr}^{-1}$ for $\nu_{\mu}\left(\bar{\nu}_{\mu}\right)$ respectively, $\theta$ is the zenith angle.

IceCube could have an angular resolution $\sim 1^{\circ}$, which is effective to suppress the diffuse atmospheric neutrino background. Notice the cluster is not an exact point source, we utilize an angular resolution of $3.0^{\circ}\left(1.5^{\circ}\right)$ for M49 (AWM47/Coma) cluster. The number of atmospheric neutrinos for $3^{\circ}$ resolution angle is $\sim 4$ times larger than it for $1.5^{\circ}$ cone.
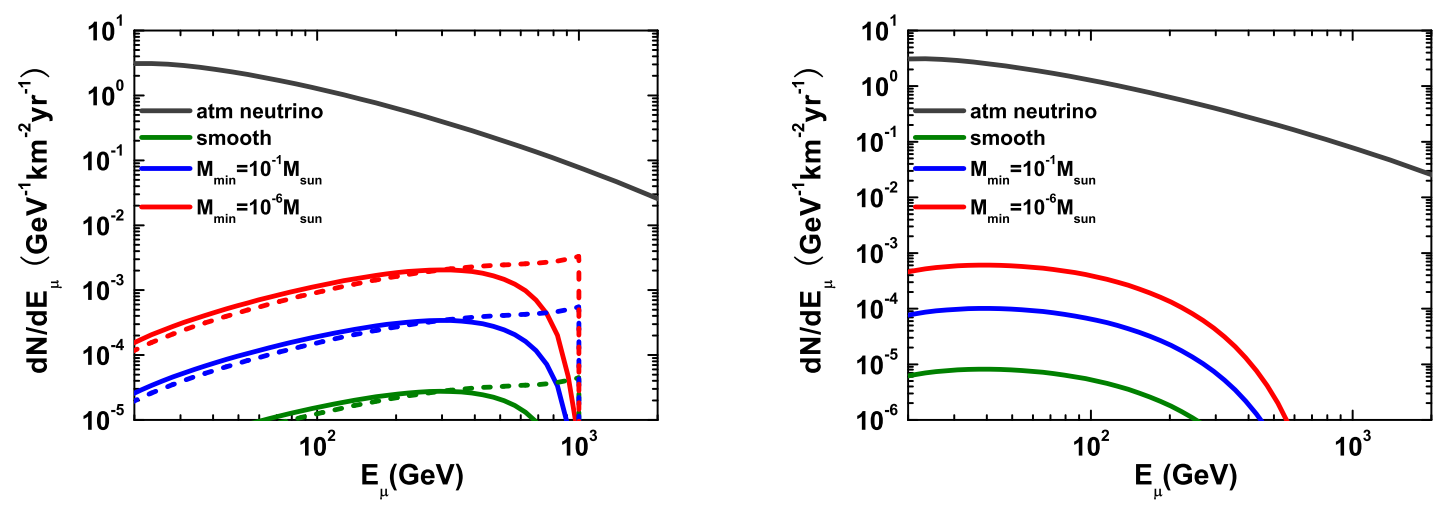

FIG. 5: Muon flux induced by neutrinos from DM annihilation in M49 cluster, for muon (left) and $b \bar{b}$ (right) final states respectively. The mass of $\mathrm{DM}$ is $1 \mathrm{TeV}$ and cross section is $10^{-23} \mathrm{~cm}^{3}$ $\mathrm{s}^{-1}$. The results of smooth halo (green) and smooth halo together with subhalos with two values of $M_{\min }, 10^{-1} \mathrm{M}_{\odot}$ (blue) and $10^{-6} \mathrm{M}_{\odot}$ (red) are shown. In the left panel, the solid lines denote $\mu^{+} \mu^{-}$channel, and dashed lines denote $\mu^{+} \mu^{-}$plus $\nu_{\mu} \bar{\nu}_{\mu}$ with equal branching ratios. Also shown is muon flux induced by atmospheric muon neutrino background in $3^{\circ}$ cone.

In Fig. 5, we show the through-going muon flux induced by DM annihilation in M49 cluster. Similar as in Fig. [1 we adopt $m_{\chi}=1 \mathrm{TeV},\langle\sigma v\rangle=10^{-23} \mathrm{~cm}^{3} \mathrm{~s}^{-1}$, and annihilation channels are $\mu^{+} \mu^{-}$(left solid), $\mu^{+} \mu^{-}+\nu_{\mu} \bar{\nu}_{\mu}\left(B_{\mu}=B_{\nu}=0.5\right.$, left dashed) and $b \bar{b}$ (right). For 
$b \bar{b}$ channel, the neutrinos are produced through decay of hadrons induced by $b \bar{b}$ hadronization. It is shown that the muon spectrum of such channel is very soft and difficult to detect given the high level of background. The case for $\mu^{+} \mu^{-}$channel seems better but the signal is still very weak. Even for $M_{\min }=10^{-6} \mathrm{M}_{\odot}$, the muon flux from DM annihilation is $\sim 100$ times smaller than the background in energy range $(200,800) \mathrm{GeV}$. Only for $\mu^{+} \mu^{-}+\nu_{\mu} \bar{\nu}_{\mu}$ the situation is better. The reason is that monochromatic neutrino spectrum is harder than other channels, and is easier to be detected. Compared with Fig. 1, it is not strange to see that the neutrino detection sensitivity would be much weaker than the $\gamma$-ray detection.
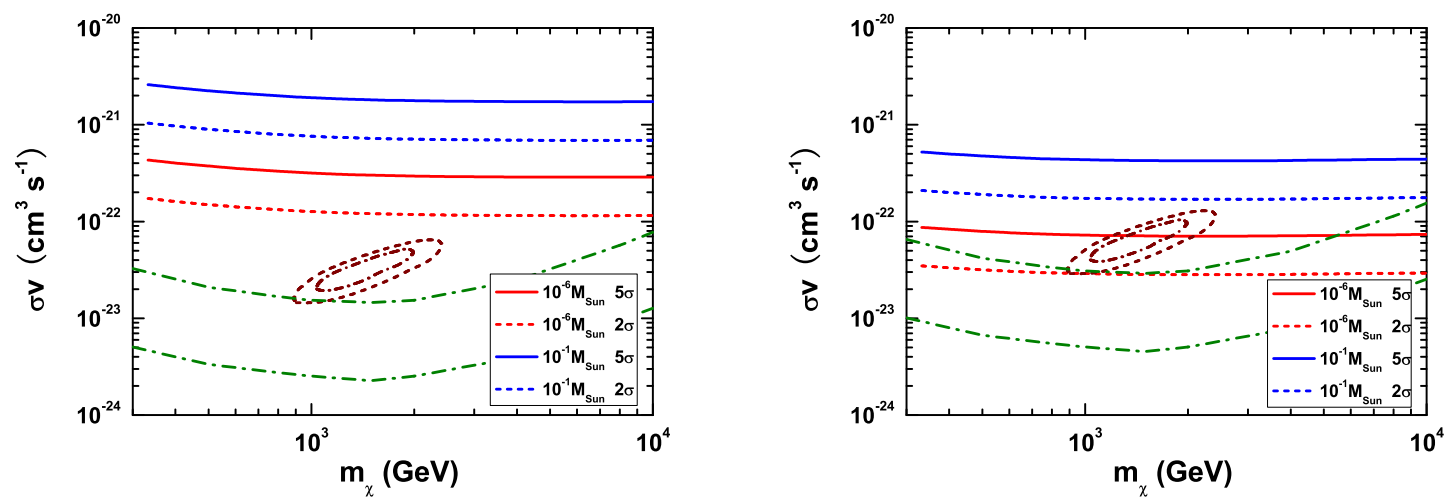

FIG. 6: $\langle\sigma v\rangle$ required to discover neutrinos from DM annihilation in M49 cluster as a function of DM mass. The left panel is for $\mu^{+} \mu^{-}$channel, and the right panel is for $\mu^{+} \mu^{-}+\nu_{\mu} \bar{\nu}_{\mu}$ with branching ratios $B_{\mu}=B_{\nu}=0.5$. Dashed circles are the $3 \sigma$ and $5 \sigma$ parameters regions which can fit the PAMELA/Fermi-LAT/HESS data of the CR positrons/electrons [56]. The dot-dashed curves represent the $\gamma$-ray constraints for $M_{\min }=10^{-1}$ and $10^{-6} \mathrm{M}_{\odot}$ respectively (see Fig. (2). The circles and $\gamma$-ray constraints in the right panel are scaled upwards by a factor 2 due to the branching ratio $B_{\mu}=0.5$.

The total muon event rate in a specific energy bin at the detector is

$$
N=\int \mathrm{d} E_{\mu} \frac{\mathrm{d} N_{\mu}}{\mathrm{d} E_{\mu}} A_{\text {eff }}\left(E_{\mu}, \theta\right) \Delta T,
$$

where $A_{\text {eff }}$ is the effective muon detecting area taken from Ref. [69], $\Delta T$ is the operation time which is set as 10 years here. We take the threshold energy to be $E_{\text {th }} \sim 40 \mathrm{GeV}$, and assume the energy resolution is $\Delta \log _{10} E=0.35[66]$. 

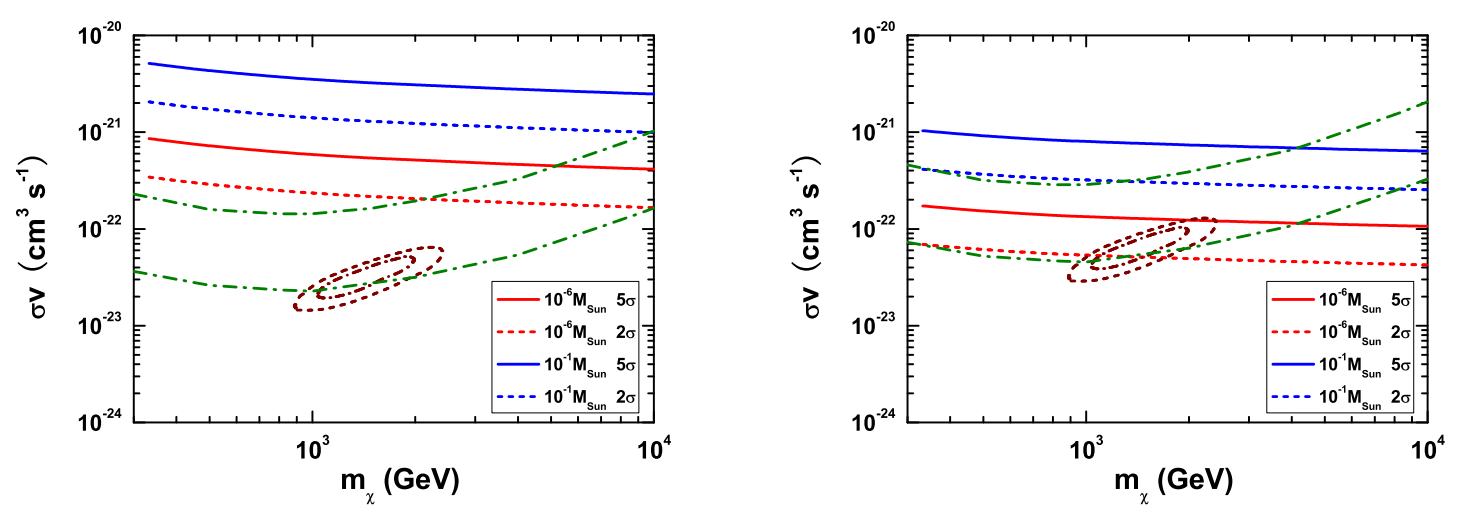

FIG. 7: The same as Fig. 6 but for AWM7 cluster.
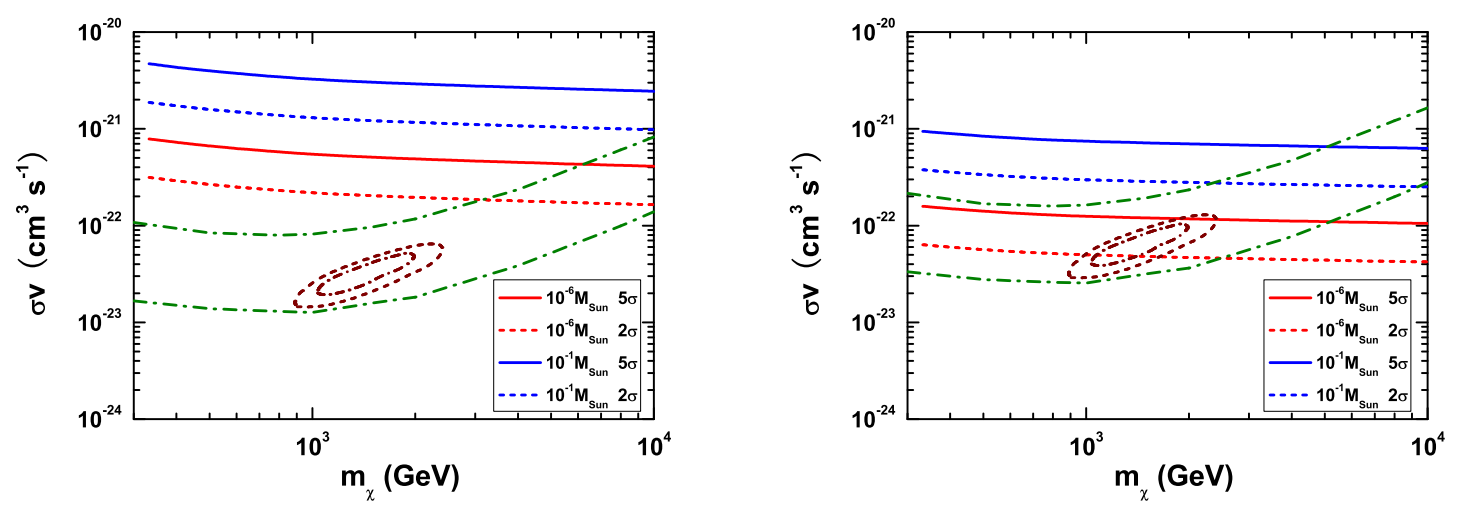

FIG. 8: The same as Fig. [6 but for Coma cluster.

In Fig. [6 we give the IceCube sensitivity of detecting neutrinos from DM annihilations in M49 for 10-yr exposure. The left and right panels show the results for annihilation channels $\mu^{+} \mu^{-}$and $\mu^{+} \mu^{-}+\nu_{\mu} \bar{\nu}_{\mu}$ respectively. For $\mu^{+} \mu^{-}$channel and $M_{\min }=10^{-6} \mathrm{M}_{\odot}$, a $5 \sigma$ detection requires $\langle\sigma v\rangle$ as large as $O\left(10^{-22}\right) \mathrm{cm}^{3} \mathrm{~s}^{-1}$. We can see such a cross section is much larger than that required to explain PAMELA/Fermi-LAT/HESS data. For the channel to equal $\mu^{+} \mu^{-}+\nu_{\mu} \bar{\nu}_{\mu}$, the sensitivity is $\sim 10$ times better than $\mu^{+} \mu^{-}$channel. Because the neutrino-nucleon cross section and muon range are approximately proportional to $E_{\nu}$, while the atmospheric neutrino background decreases as $\sim E_{\nu}^{-3}$, the neutrino telescope is more powerful to detect the high energy neutrinos. For the same reason, the detector is more 
sensitive to explore heavy DM. It is shown that if DM annihilation products have a large branching ratio to $\nu \bar{\nu}$, IceCube could reach the parameter space to explain PAMELA/FermiLAT/HESS results.

The sensitivities for other two clusters, AWM 7 and Coma, are shown in Figs. 7 and 8 respectively. These two clusters are much more distant from us than M49. Note for these two clusters we take $1.5^{\circ}$ cone, which is enough to include all of the cluster, to count the atmospheric background. Due to a much lower level of background and larger masses of AWM 7 and Coma, the sensitivities are only several tens percent weaker than M49. Because the declinations of AWM 7 and Coma are larger than M49 which is close to the horizon, it would be more effective to reject the CR muon background and could provide clearer detection of signals.

Compared with the $\gamma$-ray sensitivity discussed in Sec. II, the sensitivity of neutrino detection is relatively poor. If we employ the $\gamma$-ray constraint of DM annihilation in clusters (e.g., $M_{\min } \approx 10^{3} \mathrm{M}_{\odot}$ for PAMELA/Fermi-LAT/HESS best fit parameters), there would be little chance to detect the neutrino signals. Only for very heavy DM $\left(m_{\chi} \sim 10 \mathrm{TeV}\right)$, the sensitivities of the two ways are comparable.

\section{SUMMARY AND DISCUSSION}

In this work we investigate the $\gamma$-ray and neutrino emission from DM annihilation in clusters of galaxies. A sample of several nearby clusters is considered. Both the annihilations in the host halo and substructures are taken into account. For the annihilation luminosity of substructures we adopt the result of Aquarius simulation, and scale it from Milky Way like halo to the cluster like halo. There is also a component of unresolved substructures which is not seen due to the limit of resolution of numerical simulation. For the contribution from the unresolved substructures we adopt an extrapolation of the luminosity-mass relation fitted from the resolved subhalos in the simulation. The minimum mass of the subhalo $M_{\min }$ is left to be a free parameter. The value of $M_{\min }$ may catch the information about free streaming length, and may reflect the generation history of the DM particle.

For the $\gamma$-ray emission we consider two typical annihilation channels, quark final states $b \bar{b}$ and lepton final states $\mu^{+} \mu^{-}$. Both the primary component of photons from hadron decay and final state radiation, and the secondary component which is produced by the IC 
scattering of DM-induced electrons/positrons and CMB field are taken into account. The Fermi-LAT upper limits of the $\gamma$-ray emission from these clusters are employed to constrain the $m_{\chi}-\langle\sigma v\rangle$ parameter plane. The constraints depend on the value of $M_{\min }$. For $M_{\min }$ down to $\sim 10^{-6} \mathrm{M}_{\odot}$, which is expected for the structure formation of neutralino-like CDM picture, the constraints are $\sim 50$ times stronger than the case of smooth halo only. Typically for $m_{\chi}=1 \mathrm{TeV}$ and $M_{\min }=10^{-6} \mathrm{M}_{\odot}$ the strongest constraint on cross section from the cluster sample is $10^{-24}\left(10^{-24}\right) \mathrm{cm}^{3} \mathrm{~s}^{-1}$ for $\mu^{+} \mu^{-}(b \bar{b})$.

It is of great interest for the $\mu^{+} \mu^{-}$channel which is proposed to explain the positron and electron excesses reported by PAMELA, Fermi-LAT and HESS [56, 70]. A very large annihilation cross section (or boost factor) is needed to explain the data. It is shown that the Fermi-LAT observations about $\gamma$-rays from galaxy clusters can strongly constrain the model parameters. If we fix the mass and cross section to the values explaining the $e^{ \pm}$excesses, the minimum mass of substructures $M_{\min }$ is constrained to be larger than $10^{2}-10^{3} \mathrm{M}_{\odot}$. Such a large value of halo mass means a very large free streaming length of DM particle. It may indicate the nature of DM particles is warm instead of cold [40, 63].

Finally we calculate the sensitivity of detecting neutrinos from these clusters by the IceCube detector. It is shown to detect neutrinos would be much more difficult than $\gamma$-rays. For $b \bar{b}$ final state the sensitivity is extremely poor due to the neutrino spectrum from $b \bar{b}$ hadronization is soft and suffers from a very high atmospheric background. The case becomes better for $\mu^{+} \mu^{-}$final state. However, the signal is still very weak. For example, even for $M_{\text {min }}=10^{-6} \mathrm{M}_{\odot}$, a $5 \sigma$ detection with $\sim 10$-yr exposure requires $\langle\sigma v\rangle$ as large as $O\left(10^{-22}\right)$ $\mathrm{cm}^{3} \mathrm{~s}^{-1}$. This result does not have enough capability to explore the PAMELA/FermiLAT/HESS favored parameter region. If we consider the model with a large fraction of line neutrino emission the detectability would be much better (e.g. improved by an order of magnitude). However, compared with $\gamma$-rays the constraint from neutrinos is still some weaker. Only for very heavy DM $\left(m_{\chi} \sim 10 \mathrm{TeV}\right)$, the sensitivity of neutrino detection can be comparable with $\gamma$-rays.

\section{Acknowledgments}

This work was supported in part by the Natural Sciences Foundation of China under the grant Nos. 10773011, 10775001, 10635030, the 973 project under grant 2010CB833000, and 
the trans-century fund of Chinese Ministry of Education.

[1] G. Jungman, M. Kamionkowski, and K. Griest, Phys. Rept. 267, 195 (1996), arXiv:hep$\mathrm{ph} / 9506380$.

[2] G. Bertone, D. Hooper, and J. Silk, Phys. Rept. 405, 279 (2005), arXiv:hep-ph/0404175.

[3] O. Adriani, G. C. Barbarino, G. A. Bazilevskaya, R. Bellotti, M. Boezio, E. A. Bogomolov, L. Bonechi, M. Bongi, V. Bonvicini, S. Bottai, et al., Nature 458, 607 (2009), 0810.4995.

[4] O. Adriani, G. C. Barbarino, G. A. Bazilevskaya, R. Bellotti, M. Boezio, E. A. Bogomolov, L. Bonechi, M. Bongi, V. Bonvicini, S. Bottai, et al., Phys. Rev. Lett. 102, 051101 (2009), 0810.4994 .

[5] J. Chang, J. H. Adams, H. S. Ahn, G. L. Bashindzhagyan, M. Christl, O. Ganel, T. G. Guzik, J. Isbert, K. C. Kim, E. N. Kuznetsov, et al., Nature 456, 362 (2008).

[6] F. Aharonian, A. G. Akhperjanian, U. Barres de Almeida, A. R. Bazer-Bachi, Y. Becherini, B. Behera, W. Benbow, K. Bernlöhr, C. Boisson, A. Bochow, et al., Phys. Rev. Lett. 101, 261104 (2008), 0811.3894.

[7] F. Aharonian, A. G. Akhperjanian, G. Anton, U. Barres de Almeida, A. R. Bazer-Bachi, Y. Becherini, B. Behera, K. Bernlöhr, A. Bochow, C. Boisson, et al., Astron. Astrophys. 508, $561(2009)$.

[8] A. A. Abdo, M. Ackermann, M. Ajello, W. B. Atwood, M. Axelsson, L. Baldini, J. Ballet, G. Barbiellini, D. Bastieri, M. Battelino, et al., Phys. Rev. Lett. 102, 181101 (2009), 0905.0025.

[9] M. Cirelli, M. Kadastik, M. Raidal, and A. Strumia, Nuclear Physics B 813, 1 (2009), 0809.2409.

[10] P. F. Yin, Q. Yuan, J. Liu, J. Zhang, X. J. Bi, S. H. Zhu, and X. M. Zhang, Phys. Rev. D 79, 023512 (2009), 0811.0176.

[11] J. Liu, Q. Yuan, X. Bi, H. Li, and X. Zhang, Phys. Rev. D 81, 023516 (2010), 0906.3858.

[12] J. Liu, Q. Yuan, X. J. Bi, H. Li, and X. M. Zhang, ArXiv e-prints:0911.1002 (2009), 0911.1002.

[13] G. Bertone, M. Cirelli, A. Strumia, and M. Taoso, Journal of Cosmology and Astro-Particle Physics 3, 9 (2009), 0811.3744.

[14] J. Zhang, X. J. Bi, J. Liu, S. M. Liu, P. F. Yin, Q. Yuan, and S. H. Zhu, Phys. Rev. D 80, 
023007 (2009), 0812.0522.

[15] L. Bergström, G. Bertone, T. Bringmann, J. Edsjö, and M. Taoso, Phys. Rev. D 79, 081303 (2009), 0812.3895.

[16] J. Zhang, Q. Yuan, and X. J. Bi, ArXiv e-prints (2009), 0908.1236.

[17] M. Cirelli, P. Panci, and P. D. Serpico, ArXiv e-prints (2009), 0912.0663.

[18] L. Zhang, C. Weniger, L. Maccione, J. Redondo, and G. Sigl, ArXiv e-prints (2009), 0912.4504.

[19] C. Chen, M. M. Nojiri, S. C. Park, and J. Shu, ArXiv e-prints (2009), 0908.4317.

[20] M. Papucci and A. Strumia, Journal of Cosmology and Astro-Particle Physics 3, 14 (2010), 0912.0742 .

[21] J. Hisano, M. Kawasaki, K. Kohri, and K. Nakayama, Phys. Rev. D 79, 043516 (2009), 0812.0219 .

[22] J. Liu, P. Yin, and S. Zhu, Phys. Rev. D 79, 063522 (2009), 0812.0964.

[23] D. Spolyar, M. Buckley, K. Freese, D. Hooper, and H. Murayama, ArXiv e-prints (2009), 0905.4764 .

[24] M. R. Buckley, D. Spolyar, K. Freese, D. Hooper, and H. Murayama, Phys. Rev. D 81, 016006 (2010), 0907.2385.

[25] S. K. Mandal, M. R. Buckley, K. Freese, D. Spolyar, and H. Murayama, Phys. Rev. D 81, 043508 (2010), 0911.5188.

[26] P. Sandick, D. Spolyar, M. Buckley, K. Freese, and D. Hooper, Phys. Rev. D 81, 083506 (2010), 0912.0513.

[27] L. Covi, M. Grefe, A. Ibarra, and D. Tran, Journal of Cosmology and Astro-Particle Physics 4, 17 (2010), 0912.3521.

[28] E. Borriello, A. Cuoco, and G. Miele, Astrophys. J. Lett. 699, L59 (2009), 0903.1852.

[29] R. Essig, N. Sehgal, and L. E. Strigari, Phys. Rev. D 80, 023506 (2009), 0902.4750.

[30] L. Pieri, M. Lattanzi, and J. Silk, Mon. Not. Roy. Astron. Soc. 399, 2033 (2009), 0902.4330.

[31] M. Kuhlen, P. Madau, and J. Silk, Science 325, 970 (2009), 0907.0005.

[32] L. Pieri, J. Lavalle, G. Bertone, and E. Branchini, ArXiv e-prints (2009), 0908.0195.

[33] M. Kawasaki, K. Kohri, and K. Nakayama, Phys. Rev. D 80, 023517 (2009), 0904.3626.

[34] S. Profumo and T. E. Jeltema, Journal of Cosmology and Astro-Particle Physics 7, 20 (2009), 0906.0001.

[35] J. Zavala, V. Springel, and M. Boylan-Kolchin, Mon. Not. Roy. Astron. Soc. 405, 593 (2010), 
0908.2428 .

[36] G. Hütsi, A. Hektor, and M. Raidal, Astron. Astrophys. 505, 999 (2009), 0906.4550.

[37] M. Cirelli, F. Iocco, and P. Panci, Journal of Cosmology and Astro-Particle Physics 10, 9 (2009), 0907.0719.

[38] Q. Yuan, B. Yue, X. Bi, X. Chen, and X. Zhang, ArXiv e-prints (2009), 0912.2504.

[39] T. E. Jeltema, J. Kehayias, and S. Profumo, Phys. Rev. D 80, 023005 (2009), 0812.0597.

[40] A. Pinzke, C. Pfrommer, and L. Bergström, Phys. Rev. Lett. 103, 181302 (2009), 0905.1948.

[41] A. A. Abdo, M. Ackermann, M. Ajello, W. B. Atwood, L. Baldini, J. Ballet, G. Barbiellini, D. Bastieri, B. M. Baughman, K. Bechtol, et al., Journal of Cosmology and Astro-Particle Physics 05, 025 (2010).

[42] A. A. Abdo, M. Ackermann, M. Ajello, K. Asano, L. Baldini, J. Ballet, G. Barbiellini, D. Bastieri, B. M. Baughman, K. Bechtol, et al., Astrophys. J. 699, 31 (2009), 0904.1904.

[43] J. F. Beacom, N. F. Bell, and G. D. Mack, Physical Review Letters 99, 231301 (2007), arXiv:astro-ph/0608090.

[44] H. Yüksel, S. Horiuchi, J. F. Beacom, and S. Ando, Phys. Rev. D 76, 123506 (2007), 0707.0196.

[45] W. B. Atwood, A. A. Abdo, M. Ackermann, W. Althouse, B. Anderson, M. Axelsson, L. Baldini, J. Ballet, D. L. Band, G. Barbiellini, et al., Astrophys. J. 697, 1071 (2009), 0902.1089.

[46] D. A. Buote, F. Gastaldello, P. J. Humphrey, L. Zappacosta, J. S. Bullock, F. Brighenti, and W. G. Mathews, Astrophys. J. 664, 123 (2007), arXiv:astro-ph/0610135.

[47] W. Hu and A. V. Kravtsov, Astrophys. J. 584, 702 (2003), arXiv:astro-ph/0203169.

[48] T. H. Reiprich and H. Böhringer, Astrophys. J. 567, 716 (2002), arXiv:astro-ph/0111285.

[49] T. Sjöstrand, S. Mrenna, and P. Skands, Journal of High Energy Physics 5, 26 (2006), arXiv:hep-ph/0603175.

[50] J. F. Navarro, C. S. Frenk, and S. D. M. White, Astrophys. J. 490, 493 (1997), arXiv:astro$\mathrm{ph} / 9611107$.

[51] V. Springel, S. D. M. White, C. S. Frenk, J. F. Navarro, A. Jenkins, M. Vogelsberger, J. Wang, A. Ludlow, and A. Helmi, Nature 456, 73 (2008), 0809.0894.

[52] V. Springel, J. Wang, M. Vogelsberger, A. Ludlow, A. Jenkins, A. Helmi, J. F. Navarro, C. S. Frenk, and S. D. M. White, Mon. Not. Roy. Astron. Soc. 391, 1685 (2008), 0809.0898.

[53] Q. Yuan, Y. X. Cao, X. J. Bi, and L. Gao, in preparation (2010). 
[54] S. Hofmann, D. J. Schwarz, and H. Stöcker, Phys. Rev. D 64, 083507 (2001), arXiv:astro$\mathrm{ph} / 0104173$.

[55] S. Colafrancesco and P. Blasi, Astroparticle Physics 9, 227 (1998), arXiv:astro-ph/9804262.

[56] P. Meade, M. Papucci, A. Strumia, and T. Volansky, Nuclear Physics B 831, 178 (2010), 0905.0480.

[57] A. V. Macciò, A. A. Dutton, and F. C. van den Bosch, Mon. Not. Roy. Astron. Soc. 391, 1940 (2008), 0805.1926.

[58] M. Kuhlen, J. Diemand, and P. Madau, Astrophys. J. 686, 262 (2008), 0805.4416.

[59] J. Hisano, S. Matsumoto, and M. M. Nojiri, Physical Review Letters 92, 031303 (2004), arXiv:hep-ph/0307216.

[60] N. Arkani-Hamed, D. P. Finkbeiner, T. R. Slatyer, and N. Weiner, Phys. Rev. D 79, 015014 (2009), 0810.0713.

[61] R. A. C. Croft, D. H. Weinberg, M. Pettini, L. Hernquist, and N. Katz, Astrophys. J. 520, 1 (1999), arXiv:astro-ph/9809401.

[62] P. McDonald, U. Seljak, S. Burles, D. J. Schlegel, D. H. Weinberg, R. Cen, D. Shih, J. Schaye, D. P. Schneider, N. A. Bahcall, et al., Astrophys. J. Supp. 163, 80 (2006), arXiv:astro$\mathrm{ph} / 0405013$.

[63] W. B. Lin, D. H. Huang, X. Zhang, and R. Brandenberger, Phys. Rev. Lett. 86, 954 (2001), arXiv:astro-ph/0009003.

[64] X. Bi, M. Li, and X. Zhang, Phys. Rev. D 69, 123521 (2004), arXiv:hep-ph/0308218.

[65] X. Bi, R. Brandenberger, P. Gondolo, T. J. Li, Q. Yuan, and X. M. Zhang, Phys. Rev. D 80, 103502 (2009), 0905.1253.

[66] E. Resconi and For the IceCube Collaboration, Nuclear Instruments and Methods in Physics Research A 602, 7 (2009).

[67] A. E. Erkoca, M. H. Reno, and I. Sarcevic, Phys. Rev. D 80, 043514 (2009), 0906.4364.

[68] M. Honda, T. Kajita, K. Kasahara, S. Midorikawa, and T. Sanuki, Phys. Rev. D 75, 043006 (2007).

[69] M. C. Gonzalez-Garcia, F. Halzen, and S. Mohapatra, Astroparticle Physics 31, 437 (2009), 0902.1176 .

[70] L. Bergström, J. Edsjö, and G. Zaharijas, Physical Review Letters 103, 031103 (2009), 0905.0333. 